\title{
Context-Dependent Responses of Primate Nucleus Basalis Neurons in a Go/No-Go Task
}

\author{
Russell T. Richardson ${ }^{1}$ and Mahlon R. DeLong ${ }^{1,2}$ \\ Departments of ${ }^{1}$ Neurology and ${ }^{2}$ Neuroscience, Johns Hopkins University School of Medicine, Baltimore, Maryland 21205
}

In previous studies involving monkeys performing behavioral tasks, neurons in the nucleus basalis frequently had significant changes in discharge rate when the animal made a movement in response to a sensory stimulus in order to obtain a reward. To determine whether such responses of basalis neurons are primarily sensory or motor in nature, the activity of single basalis neurons was recorded in monkeys performing a go/no-go (GNG) task which provided a dissociation between sensory and motor neuronal responses. In a sample of 425 basalis neurons, $326(77 \%)$ had significant changes in firing in at least one phase of the GNG task. Most of the task-related neurons $(70 \%)$ responded in the choice phase in which the animal either made an arm movement (go condition) or kept its arm motionless (no-go condition) in order to obtain a water reward. Of 253 neurons that responded in the choice phase, $88 \%$ had changes in firing in the no-go condition that were equal to or, in some cases, greater than the changes in firing in the go condition. Therefore, most responses of basalis neurons in the choice phase could not be specific for the arm movement because they occurred when there was no arm movement at all.

The visual stimulus presented in the choice phase was also presented earlier on each trial in the cue phase. Although $70 \%$ of the task-related basalis neurons responded in the choice phase, only $5 \%$ had detectable changes in firing in the cue phase. Of 251 neurons responding in the cue or choice phase, $59 \%$ had significantly larger changes in firing in the choice phase than in the cue phase, whereas only one neuron had a larger response in the cue phase. Therefore, most responses of basalis neurons in the choice phase could not be specific for the visual stimulus because similar responses did not occur when the same stimulus was presented in the cue phase. These results indicate that the frequent responses of basalis neurons in the choice phase are neither purely sensory nor motor in nature, but are highly dependent on the context of the stimulus or movement. The neuronal responses in the choice phase may reflect either transient increases in arousal or decision-making processes.

\footnotetext{
Received July 17, 1989; revised Feb. 8, 1990; accepted Feb. 26, 1990.

Wc wish to cxpress our apprcciation of Nancy Derevjanik and Lisa Frecman for technical support, Frank Baker and Ross Cutler for computer programming, and Garrett Alexander, Michael Crutcher, Apostolos Georgopoulos, and David Olton for comments on the manuscript. This work was supported by NIH Grant 2 P50 NS 20471.

Correspondence should be addressed to Dr. Russell T. Richardson, Department of Neurology, Meyer 5-185, Johns Hopkins School of Medicine, 600 North Wolfe Street, Baltimore, MD 21205.
}

Copyright (C) 1990 Society for Neuroscience $0270-6474 / 90 / 082528-13 \$ 03.00 / 0$
The nucleus basalis of Meynert is the primary source of cholinergic innervation of cerebral cortex in primates (Mesulam et al., 1983; Struble et al., 1986) and may therefore have a substantial influence on cortical functions. It has been hypothesized to be involved in a number of processes, including arousal, learning, and memory (see Richardson and DeLong, 1988), but there have been few studies attempting to demonstrate whether neuronal activity in the nucleus basalis is compatible with these processes. Previous studies in behaving monkeys have indicated that changes in neuronal activity in the nucleus basalis may be associated with limb movements (Mitchell et al., 1987) as well as the delivery of a water reward (DeLong, 1971). Basalis neurons have also been found to be particularly responsive to stimuli that precede a reward. In a previous study, numerous basalis neurons had large changes in discharge rate in response to a brief torque applied to the arm that was consistently followed by a water reward, but had substantially smaller responses to a sustained torque that was not followed by a water reward (Mitchell et al., 1987; Richardson et al., 1988). Likewise, in a delayed response (DR) task, almost $2 / 3$ of all task-related basalis neurons responded when the monkey moved a cursor to a target in order to obtain a water reward, but fewer than $1 / 3$ of the neurons responded when the monkey made the same movement to the same target earlier in the trial (Richardson and DeLong, 1986b).

These findings suggested that the responses of basalis neurons were neither purely sensory nor motor in nature since they varied considerably under different conditions. It was not possible, however, to make such a distinction using the previous tasks. Therefore, a go/no-go (GNG) task has been employed to determine whether responses of basalis neurons could be related to specific sensory stimuli or specific movements.

We recorded the activity of single neurons in the nucleus basalis of monkeys as they performed the GNG task. Neuronal activity during one part of the task was compared to aclivity in other parts to determine precisely which components of the task elicited responses in basalis neurons. Neuronal activity in the go condition (when the animal made a specific arm movement) was compared to activity in the no-go condition (when no movement was made) to determine whether the movement was a necessary condition for responses of basalis neurons. Similarly, neuronal activity following a specific sensory stimulus early in the trial was compared to activity following the same stimulus presented later in the trial to determine whether the basalis neuronal responses were specific for a particular visual stimulus. It was therefore possible to establish whether responses of basalis neurons could be characterized as primarily sensory or motor in nature, or neither.

Another issue addressed by this study was whether the activity of basalis neurons could be related to the memory component 


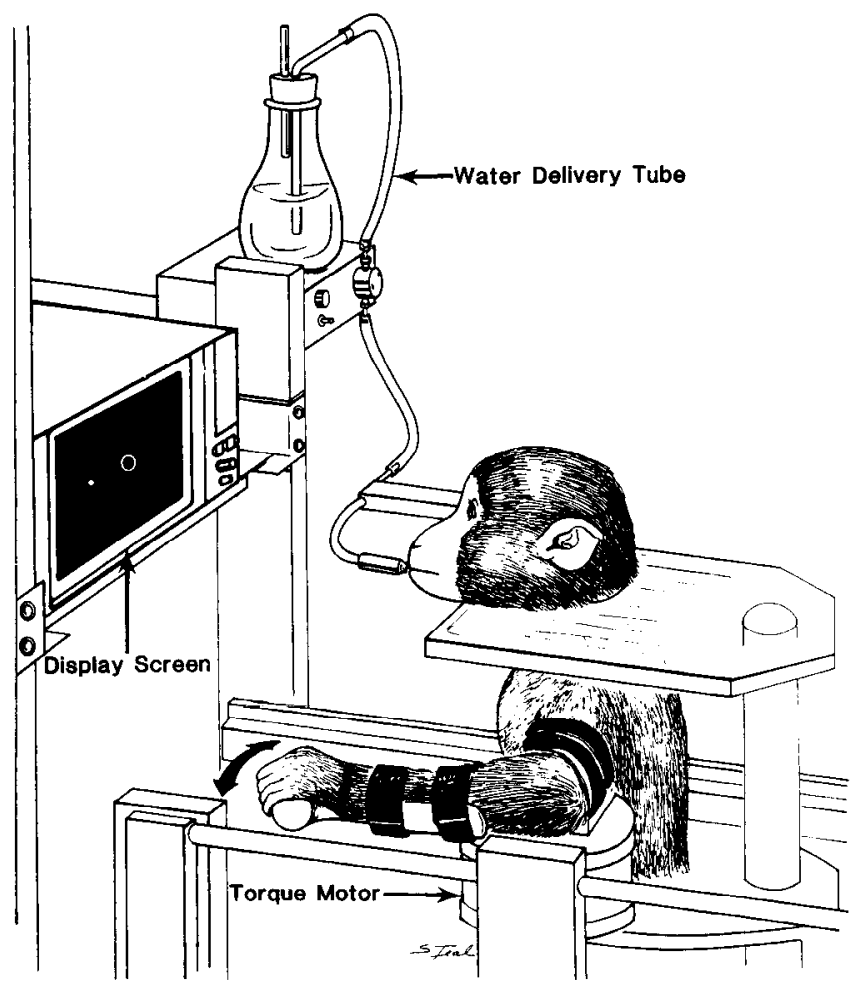

Figure 1. Behavioral apparatus. The monkey sat in a primate chair with one arm attached to a torque motor handle. Movements of the handle moved a small dot on the oscilloscope screen in front of the animal. The monkey had to keep the dot within the circle displayed on the screen throughout most of the trial. Correct execution of each trial was rewarded with a drop of water delivered through a tube at the animal's mouth.

of a behavioral task. In the GNG task, as in the previous DR task, the monkey was required to remember the position of a visual stimulus during a delay period of several seconds. During such delay periods, neurons in the dorsolateral prefrontal cortex have been shown to have robust, differential responses, which have been interpreted to be related to the memory component of the DR task (Fuster, 1973; Niki and Watanabe, 1976; Kojima and Goldman-Rakic, 1982). In contrast, basalis neurons have shown little evidence of having similar delay period responses in the DR task (Richardson and DeLong, 1986b). Since it has often been suggested that the nucleus basalis is directly involved in memory processes (Collerton, 1986; Olton and Wenk, 1987), it is important to determine whether basalis neurons have changes in discharge rates during the delay period that could possibly reflect the information being remembered by the animal.

Preliminary results of this study have been presented previously (Richardson and DeLong, 1986a; Richardson et al., 1988).

\section{Materials and Methods}

Two juvenile male rhesus monkeys (Macaca mulatta) were trained to perform the GNG task. After the animals became proficient on the task, the activity of single neurons within the nucleus basalis was recorded as the animals performed the task. Recordings were made in both hemispheres of the first animal and in one hemisphere of the second animal. The animals were initially trained to use one arm to perform the task, and several months later they were trained to use the opposite arm. In both animals, neuronal activity was recorded in the nucleus basalis while the animal used the contralateral arm or the ipsilateral arm to perform the task.
Behavioral task. To perform the GNG task, the monkey sat in a primate chair with one arm on a handle that moved in the horizontal plane, as shown in Figure 1. By moving the handle back and forth with flexion or extension movements about the elbow, the monkey controlled the position of a small dot on an oscilloscope screen placed $0.5 \mathrm{~m}$ in front of him. Also displayed on the screen was a $1 \mathrm{~cm}$ diameter circle whose position was controlled by the laboratory computer (PDP 11/ 23). The circle could only appear on the left, right, or center of the screen, whereas the dot (controlled by the monkey) could be positioned anywhere along the horizontal axis of the screen.

Between trials, the dot and circle were not displayed. A trial began when the circle appeared in the center of the scrcen and the monkcy positioned the dot within the circle, as shown in Figure 2. After 1.5-2 $\mathrm{sec}$, a torque pulse of $150 \mathrm{gm}$ was applied for $10 \mathrm{msec}$ to the handle. The torque moved the arm out of the center position briefly and served as a signal for the upcoming cue. After another $1.5-2 \mathrm{sec}$, a second circle appeared for 1.5-2 sec on the left or right side of the screen in a pseudorandom sequence. This part of the trial was referred to as the "cue" phase. The monkey did not move the dot out of the center circle, but was required to remember the position of the side circle during the subsequent delay period of 4-6 sec.

At the end of the delay period, the center circle extinguished as another circle appeared on either the left or right side of the screen in a pseudorandom sequence. This part of the trial was termed the "choice" phase because the animal had to choose whether or not to move his arm based on the remembered position of the side circle in the cue phase of the trial. If the circle in the choice phase was on the same side of the screen as it was in the cue phase, a go response was required in that the monkey had to move the dot to the circle within $650 \mathrm{msec}$. If the monkey held the dot in the side circle for $1.5-2 \mathrm{sec}$, it received a reward of at least $0.1 \mathrm{ml}$ of water delivered through a tube placed in front of its mouth. If the circle in the choice phase was opposite the circle in the cue phase, a no-go response was required in that the monkey had to keep the dot in the center circle for 1.5-2 sec. Correct no-go responses were also rewarded with $0.1 \mathrm{ml}$ of water. After $1.5-2 \mathrm{sec}$, the circle and dot were extinguished for a $3 \mathrm{sec}$ intertrial interval.

Given that the circle could appear on the left or on the right in the cue phase and in the choice phase, 4 trial types were possible: 2 types of go trials, in which the side circle was on the left or the right in both the cue and the choice phase, and 2 types of no-go trials, in which the side circle was on the left in the cue phase and on the right in the choice phase, or vice versa. Each of the 4 trial types was presented in a pseudorandom sequence.

If the monkey moved the dot out of the center circle or if he failed to respond in the go condition, the trial terminated, and a new trial began $3 \mathrm{sec}$ later. Both animals became very proficient on the task and made errors on approximately $10 \%$ of the 40 trails in a data collection run. On recording days, the animals received water only during the task, but they were allowed to work for as much water as they desired. The animals completed up to 1000 trials per day, which made it possible to record from several neurons in a single recording session. The animals' weights and behaviors were monitored daily to ensure that they were receiving adequate fluids.

After a monkey showed stable performance on the task, EMG activity was recorded acutely through nichrome wires inserted into the primary muscles involved in generating limb movements. As the animal performed the task, EMG activity was recorded differentially, rectified, and integrated over $10 \mathrm{msec}$ periods before being stored on the laboratory computer for later analysis. Histograms of EMG activity were used to determine whether covert muscle activation occurred when the arm was motionless in the cue, delay, and no-go conditions of the task.

Surgical procedures. Surgery was performed using sterile techniques with the animal under sodium pentobarbital anesthesia. The skull was exposed and an $18 \mathrm{~mm}$ diameter trephine hole was made in one hemisphere. A stainless steel cylinder was cemented over the hole at a $40^{\circ}$ angle to the sagittal plane with the center axis of the cylinder aligned with the approximate center of the nucleus basalis. Hardware used to hold the animal's head stationary during recording was also cemented to the skull. The cylinder was filled with an antibiotic solution and sealed with a removable cap.

Data collection and analysis. During recording sessions, the cylinder cap was removed and a Narishige MO-95 microdrive was attached to the cylinder. A glass-coated platinum-iridium electrode $(0.2-2.0 \mathrm{M} \Omega$ at $1 \mathrm{kHz}$ ) was lowered by the microdrive to the region of the nucleus basalis. After the action potentials of a neuron were isolated, each spike 


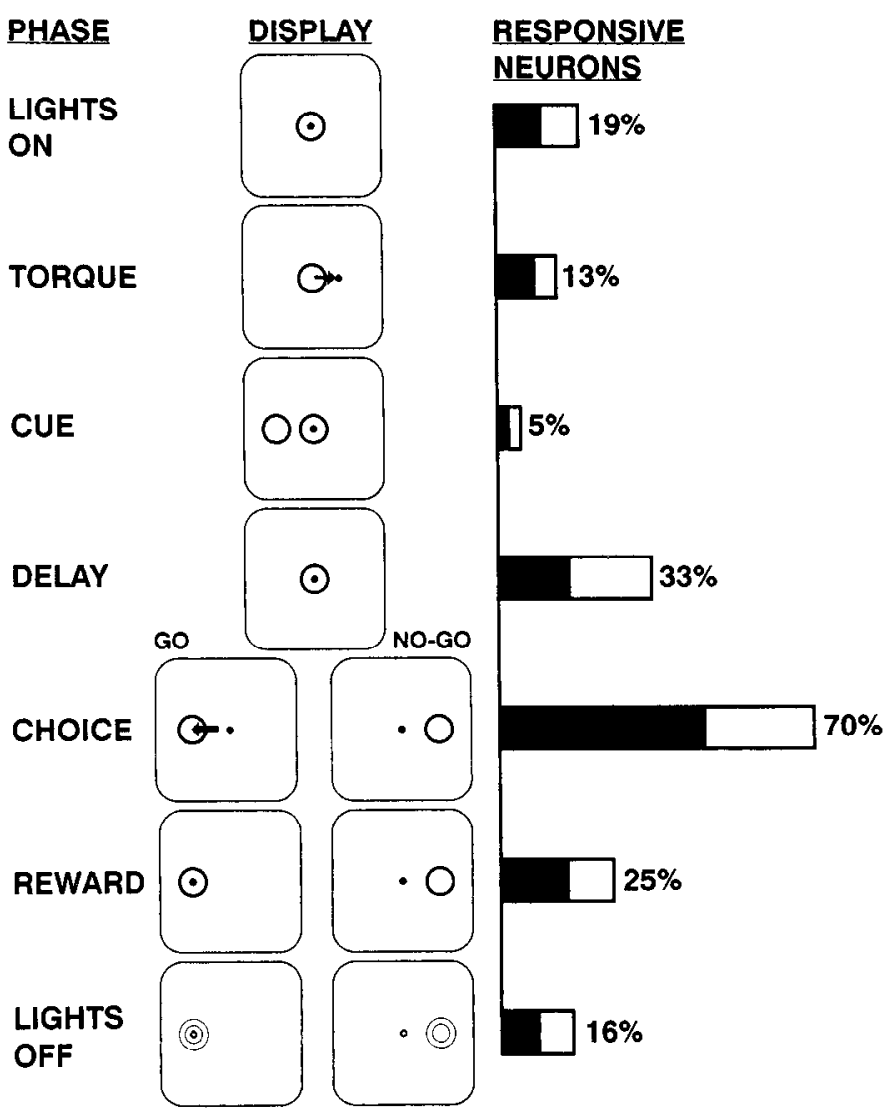

Figure 2. Task description. Each trial was composed of the 7 phases listed in the left column. The animal's view of the display screen in each phase is in the middle column. See the text for a full description of the task. In this example, the side circle in the cue phase is on the left. In the choice phase, if the side circle again appears on the left, the monkey must move the dot to the circle to obtain a reward, as indicated by the arrow. If the circle appears on the opposite side, the monkey must not move from the center position to obtain a reward. The percentage of the 326 task-related neurons that had a significant change in discharge rate in the initial $500 \mathrm{msec}$ period of each phase is shown in the right column. The solid part of each bar represents neurons with increases in discharge rate, the open part represents decreases in discharge rate.

occurrence was recorded for up to 40 trials of the task (10 samples of the 4 trial types). Data files were stored on the PDP 11/23 computer and later analyzed on a VAX system. After data collection was completed, the electrode was advanced to the next isolated neuron, and the data collection process was repeated. The locations of selected neurons werc marked by passing a $5 \mu \mathrm{A}$ DC current through the electrode at the end of some recording sessions.

The data file for a neuron contained the times at which action potentials and various stimuli occurred on each trial. As shown in Figure 2 , trials were divided into 7 phases that were delimited by the following events: (1) onset of the center circle and dot, (2) onset of the torque pulse, (3) onset of the side circle in the cue phase, (4) offset of the side circle to begin the delay period, (5) onset of the side circle in the choice phase, (6) onset of the reward delivery, and (7) offset of the dot and circle. Neuronal activity was analyzed before and after each of these events, and rasters and histograms of the recorded action potentials were constructed around each event to detect any changes in the neuron's discharge rate. Only trials on which no errors occurred were included in statistical data analyses.

On cach trial, the neuron's discharge rate in the $500 \mathrm{msec}$ period before an event (prestimulus period) was compared to the discharge in the $500 \mathrm{msec}$ period after the event (poststimulus period). A neuronal response was defined statistically as a significant $(p<0.01$, paired $t$-test) increase or decrease in the discharge rate in the poststimulus period comparcd to the prestimulus period. For the delay period, the neuron's discharge rate in the final $500 \mathrm{msec}$ of the delay period was compared to the rate in the $500 \mathrm{msec}$ period before the cue phase.

Comparisons were also made between the magnitudes of neuronal responses in different conditions of the task. The response magnitude was defined as the discharge rate in the $500 \mathrm{msec}$ poststimulus period minus the discharge rate in the $500 \mathrm{msec}$ prestimulus period. A oneway analysis of variance (ANOVA) was used to determine whether the changes in a neuron's activity in one part of the task were statistically different from its changes in activity in another part of the task.

If a neuronal response was detected by comparison of discharge rates before and after an event, then the onset, offset, and duration of the response were determined. The latency to the onset of a neuronal response was based on the instantaneous frequency (inverse of the interspike interval) collapsed across all trials. The latency was defincd as the time at which the instantaneous frequency in the poststimulus period exceeded that of the prestimulus period by more than 2 standard deviations (of the prestimulus period activity) for more than $50 \mathrm{msec}$. The end of the response was defined as the time at which the instantaneous frequency, after being more than 2 standard deviations above the prestimulus instantaneous frequency, fell below 2 standard deviations for more than $50 \mathrm{msec}$. The response duration was simply the time between the response onset and end.

Neurons were selected for analysis if they were located within the anatomical boundaries of the nucleus basalis and they had spontaneous discharge properties characteristic of basalis neurons (DeLong, 1971; Mitchell et al., 1987; Richardson and DeLong, 1987). Neurons selected for analysis had to meet 2 of the following 3 criteria: (1) a spontaneous discharge rate in the intertrial interval from 5 to $40 \mathrm{~Hz}$ (calculated as the inverse of the mean interspike interval); (2) a coefficient of variation of the interspike interval less than $100 \%$; and (3) a spike duration more than $180 \mu \mathrm{sec}$ (initial negative phase, $200-10 \mathrm{k} \mathrm{Hz}$ filtering). In the group of neurons selected for analysis in the current study, $88 \%$ met all 3 of these criteria.

Histological examination. At the end of the study, the animals were deeply anesthetized and perfused transcardially with saline followed by formalin. The brains were then sectioned and stained with cresyl violet. The sites of all recorded neurons were determined by matching electrode tracks and marker lesions with detailed records of locations of individual neurons along each electrode penetration.

\section{Results}

EMG activity

EMG activity was recorded from selected muscles of the upper arm at various times as the animal performed the task. In the go condition, elbow flexion was associated with contraction of the biceps (long head), brachialis, and brachioradialis, and elbow extension was associated with contraction of the triceps (long and lateral heads) (see Fig. 3). In the no-go condition, elbow flexors and extensors were silent, except for infrequent, brief contractions in the muscles that would be used if the monkey were to move the dot to the side circle. In such cases, the EMG activity was of a small magnitude, and there was minimal, if any, detectable movement of the arm. There was no evidence of increased EMG activity during the cue and delay phases of the task.

In the first animal, EMG recordings were also made from the semispinalis capitis muscle. This neck extensor became increasingly active prior to the delivery of the water reward as the animal extended its tongue and head toward the water spout. Although the monkey made head extension movements in both go and no-go conditions, the latency of the increase in EMG activity (measured from the start of the choice phase) was approximately $1000 \mathrm{msec}$ on no-go trials and $1500 \mathrm{msec}$ on go trials, as shown in Figure 3. This difference in latency most likely resulted from the additional time required for the execution of the arm movement on go trials, which made the reward delivery occur later than on no-go trials. In fact, the increase in 


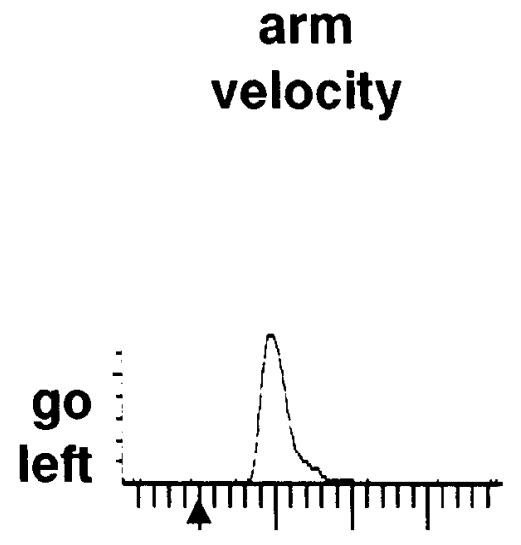

\section{brachioradialis lateral triceps}

\section{semispinalis capitis}
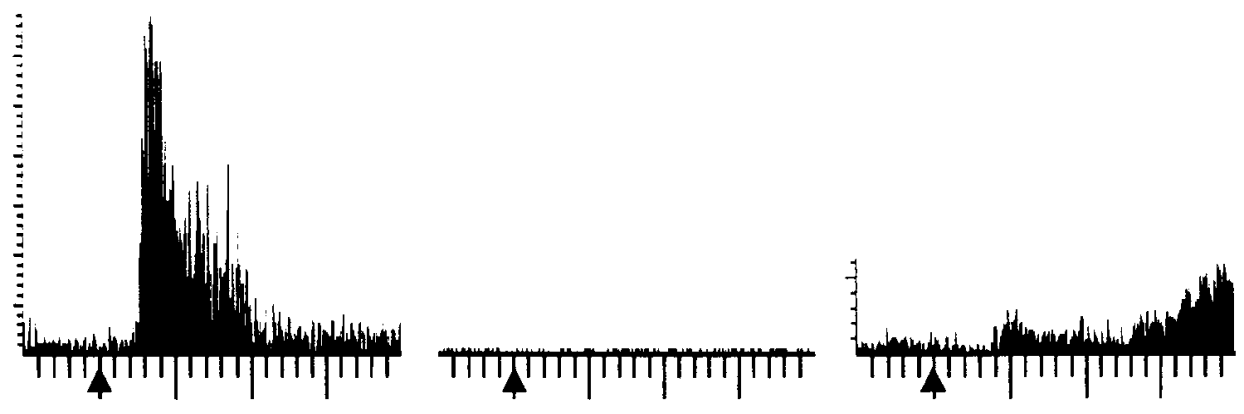

\section{no-go \\ left}
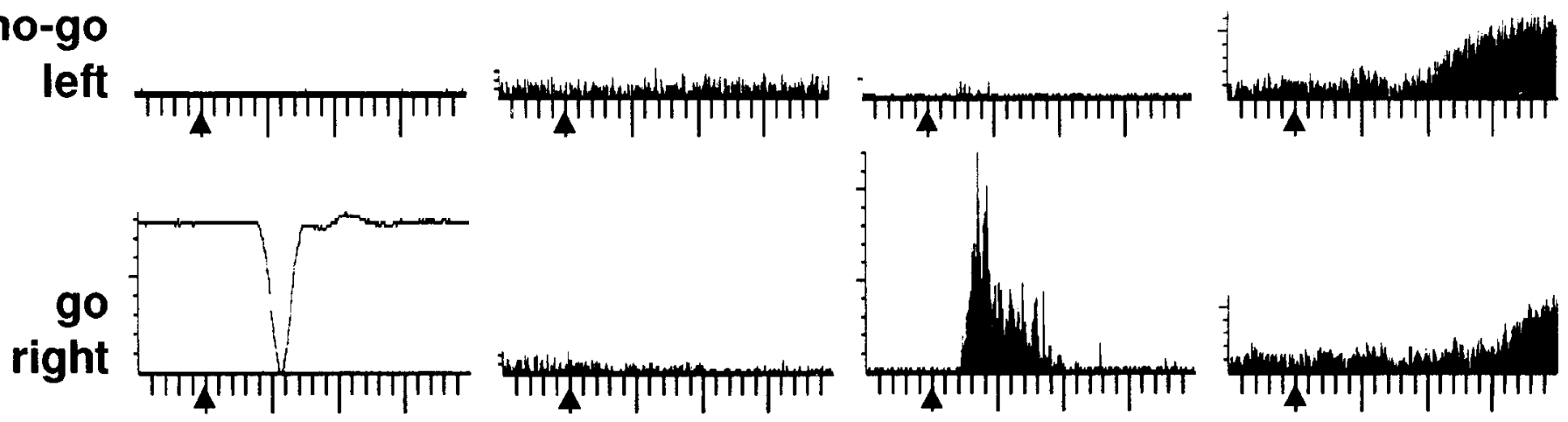

\section{no-go right}
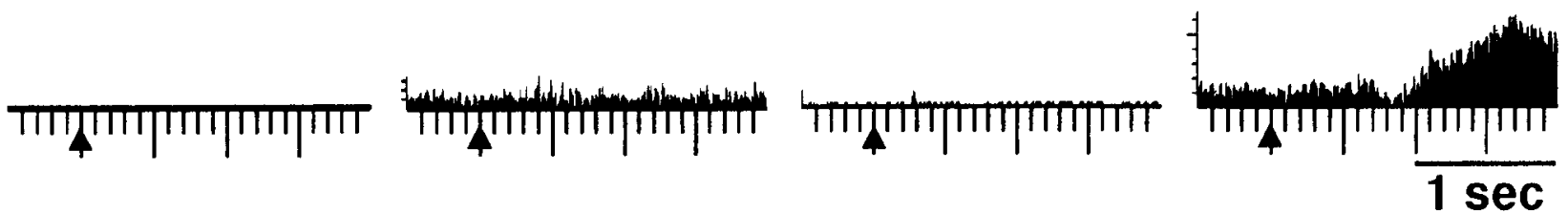

Figure 3. EMG activity in the choice phase. There were 4 possible combinations of stimuli in the cuc and choice phascs. For "go left," both the cue and the choice circles appeared on the left, and for "go right," both circles appeared on the right. For "no-go left," the cue circle was on the left, but the choice circle was on the right. For "no-go right," the cue circle was on the right, but the choice circle was on the left. All traces were from the first animal using his right arm, so "go left" required elbow flexion and "go right" required elbow extension. The arm velocity column shows the average velocity trace for 10 trials. EMG activity in the brachioradialis increased only with elbow flexion in the "go left" condition, and EMG activity in the lateral triceps increased only with elbow extension in the "go right" condition. EMG activity increased in the semispinalis capitis as the animal tried to extend its head to the water spout for the reward delivery on each correct trial. Each trace is 2.5 sec long, and the solid triangle indicates when the side circle appeared at the beginning of the choice phase. The vertical scale is in arbitrary units for EMG activity and $5.5 \% \mathrm{sec} /$ division for the velocity traces.

EMG activity in the neck relative to the time of reward delivery was the same on go trials and no-go trials, occurring approximately $800 \mathrm{msec}$ prior to the reward delivery.

\section{Database}

A total of 425 neurons were classified as nucleus basalis neurons by the criteria described in Materials and Methods. The mean firing rate of these neurons was 20 spikes $/ \mathrm{sec}(\mathrm{SD}=10.5)$, the mean coefficient of variation of the interspike interval was $64 \%$ $(\mathrm{SD}=17.0)$, and the mean spike duration was $235 \mu \mathrm{sec}(\mathrm{SD}=$
42.6) (see Table 1). In total, 193 neurons were recorded while the task was performed with the contralateral arm, and 232 neurons were recorded with the ipsilateral arm. Neurons were sampled from all major divisions of the nucleus basalis as described by Mesulam et al. (1983, 1986). In total, 97 neurons were located in the anteromedial region of the nucleus basalis, 87 were in the anterolateral region, 116 were in the intermediate region, 68 were in the posterior region, and 57 were in the medullary laminae or around the anterior commissure corresponding to the "border" neurons reported by DeLong (1971). 
Table 1. Regional differences in the nucleus basalis

\begin{tabular}{lclll} 
& & $\begin{array}{l}\text { Spon- } \\
\text { taneous } \\
\text { discharge } \\
\text { rate } \\
\text { (spikes/ } \\
\text { sec) }\end{array}$ & $\begin{array}{l}\text { Interspike } \\
\text { interval } \\
\text { coefficient } \\
\text { of varia- } \\
\text { tion }(\%)\end{array}$ & $\begin{array}{l}\text { Spike } \\
\text { duration } \\
(\mu \text { sec) }\end{array}$ \\
\hline Division & $\begin{array}{l}\text { Sample } \\
\text { size }\end{array}$ & 17.5 & 61.5 & 240 \\
Anteromedial & 97 & 19.1 & 63.4 & 233 \\
Anterolateral & 87 & 20.6 & 63.8 & 233 \\
Intermediate & 116 & 22.0 & 61.2 & 240 \\
Posterior & 68 & $\underline{24.1}$ & $\underline{71.1}$ & $\underline{226}$ \\
Rorder & 57 & 20.3 & 63.7 & 235 \\
All basalis & $\mathbf{4 2 5}$ & & & \\
\hline
\end{tabular}

\section{Neuronal responses}

For each basalis neuron, it was determined whether there was a significant change in discharge rate in any phase of the GNG task. Of the 425 neurons studied, $99(23 \%)$ showed no change in discharge rate in any phase of the task. For the remaining 326 task-related neurons, the distribution of responses among the 7 phases is shown in Figure 2. Most of the neurons $(70 \%)$ showed responses in the choice phase of the task, when the animal had to choose between moving or not moving its arm in order to obtain a reward. The fewest responses occurred in the cue phase, in which only $5 \%$ of the task-related basalis neurons had changes in firing rate. A similar pattern was found in the response magnitudes (poststimulus discharge rate minus prestimulus discharge rate) in the different phases of the task. As shown in Table 2, the neuronal responses in the choice phase had the largest median response magnitude (5.4 spikes/sec) whereas the responses in the cue phase were among the smallest (3.8 spikes/sec).

Table 2 also displays the median latencies, durations, and ends of the neuronal responses in all phases of the task. The earliest responses were in the torque phase $(74 \mathrm{msec})$ and the latest were, of course, in the delay period $(2959 \mathrm{mscc})$. In the other phases, onset latencies ranged from $124 \mathrm{msec}$ in the choice phase to $200 \mathrm{msec}$ in the reward phase. Neuronal responses in the torque phase were not only the earliest, they were also the shortest in duration $(215 \mathrm{msec})$. The median response durations in the other phases were under 350 msec except for those in the choice phase $(478 \mathrm{msec})$ and the delay period $(1779 \mathrm{msec})$. The latest median lime at which responses ended (excluding the delay period) was $611 \mathrm{msec}$ in the choice phase. Since each phase of the task was at least $1.5 \mathrm{sec}$ long, neuronal responses in one phase rarely affected the analysis of neuronal activity in the next phase.

Further analysis indicated that there was little association among neuronal responses in different phases of the task. Correlation coefficients were calculated for every possible pair of phases in the task to determine whether the presence of a neuronal response in onc phase was associated with the presence (or absence) of a response in another phase. Correlations were based only on the presence or absence of a neuronal response regardless of its magnitude or polarity. Since the neuronal responses typically ended before the beginning of the next phase, there was little chance that the detection of a neuronal response in one phase was contaminated by the presence of a response
Table 2. Neuronal response profiles ${ }^{\prime \prime}$

\begin{tabular}{llccc} 
Phase & $\begin{array}{l}\text { Magnitude }(n)^{b} \text { Latency }(n) \\
(\text { spikes/sec) }\end{array}$ & $\begin{array}{l}\text { Duration }(n) \\
(\mathrm{msec})\end{array}$ & $\begin{array}{l}\text { End }(n) \\
(\mathrm{msec})\end{array}$ \\
\hline Lights on & $4.0(63)$ & $132(9)$ & $302(8)$ & $523(8)$ \\
Torque & $3.4(40)$ & $74(27)$ & $215(27)$ & $399(27)$ \\
Cue & $3.8(17)$ & $127(10)$ & $263(10)$ & $420(10)$ \\
Delay & $4.3(106)$ & $2959(78)$ & $1779(72)$ & $4686(72)$ \\
Choice & $5.4(228)$ & $124(214)$ & $478(167)$ & $611(167)$ \\
Reward & $4.3(80)$ & $200(72)$ & $327(44)$ & $537(44)$ \\
Lights off & $3.6(50)$ & $167(38)$ & $233(26)$ & $387(26)$ \\
\hline
\end{tabular}

all entries are median values.

${ }^{b}$ The response magnitude did not represent the largest change in firing because the neuronal response began after the start of the $500 \mathrm{msec}$ poststimulus period and often continued beyond the end of the period. Nevertheless, the paired $t$-test comparisons between discharge rates in the prestimulus and poststimulus periods were quite sensitive and, consequently, were able to detect responses for which the onset could not be determined by the 2 standard deviation criterion (see Materials and Methods). Hence, the number of responses $(n)$ for each entry can vary considerably within a row.

in the preceding phase. The samples for the correlations were all task-related neurons, and the estimates of variance and covariance were those described in Kimball (1987). Significant correlations $(d f=324, p<0.01)$ existed between the following pairs of phases (refer to Fig. 2): lights on and cue $(r=0.188)$, delay and choice $(r=-0.270)$, choice and reward $(r=-0.204)$, and reward and lights off $(r=-0.157)$. All of these correlations were quite small, suggesting that a neuronal response in one phase of the task had no more than a weak association with responses in other phases.

The responses in the various phases of the task were not completely independent of each other, however, because the polarity (increase or decrease) of the response in one phase often corresponded to the polarity of the response in another phase. A chi-square analysis showed that there was a significantly higher number of neurons responding with the same polarity of response in 2 or more phases of the task than would have been predicted by chance $\left(\chi^{2}=18.9, d f=2, p<0.001\right)$. Over half $(56 \%)$ of the task-related neurons responded in 2 or more phases of the task, including $23 \%$ that responded in 3 or more phases.

\section{Go vs. no-go responses}

Neuronal responses in the choice phase were analyzed further to detect any differences between go trials and no-go trials. The magnitude of the neuronal response (see Materials and Methods) in the go condition was compared to the no-go condition in 253 basalis neurons that had responses in the choice phase. (The sample included 10 neurons that had significant changes in firing only in the go condition and 12 neurons with changes only in the no-go condition.) Significant $(p<0.01)$ differences in response magnitude were present in 52 neurons $(21 \%)$, thus indicating that $201(79 \%)$ of the 253 basalis neurons showed essentially the same change in activity in both the go and no-go conditions. Of the 52 neurons that had different responses, 30 had larger changes in activity in the go condition, and 22 had larger changes in the no-go condition. Hence, $88 \%(201+22)$ of the 253 basalis neurons had responses in the no-go condition that were equal to or greater than the responses in the go condition. Figures 4 and 5 show examples of activity from basalis neurons that had equivalent changes in firing rate in the go and no-go conditions. 


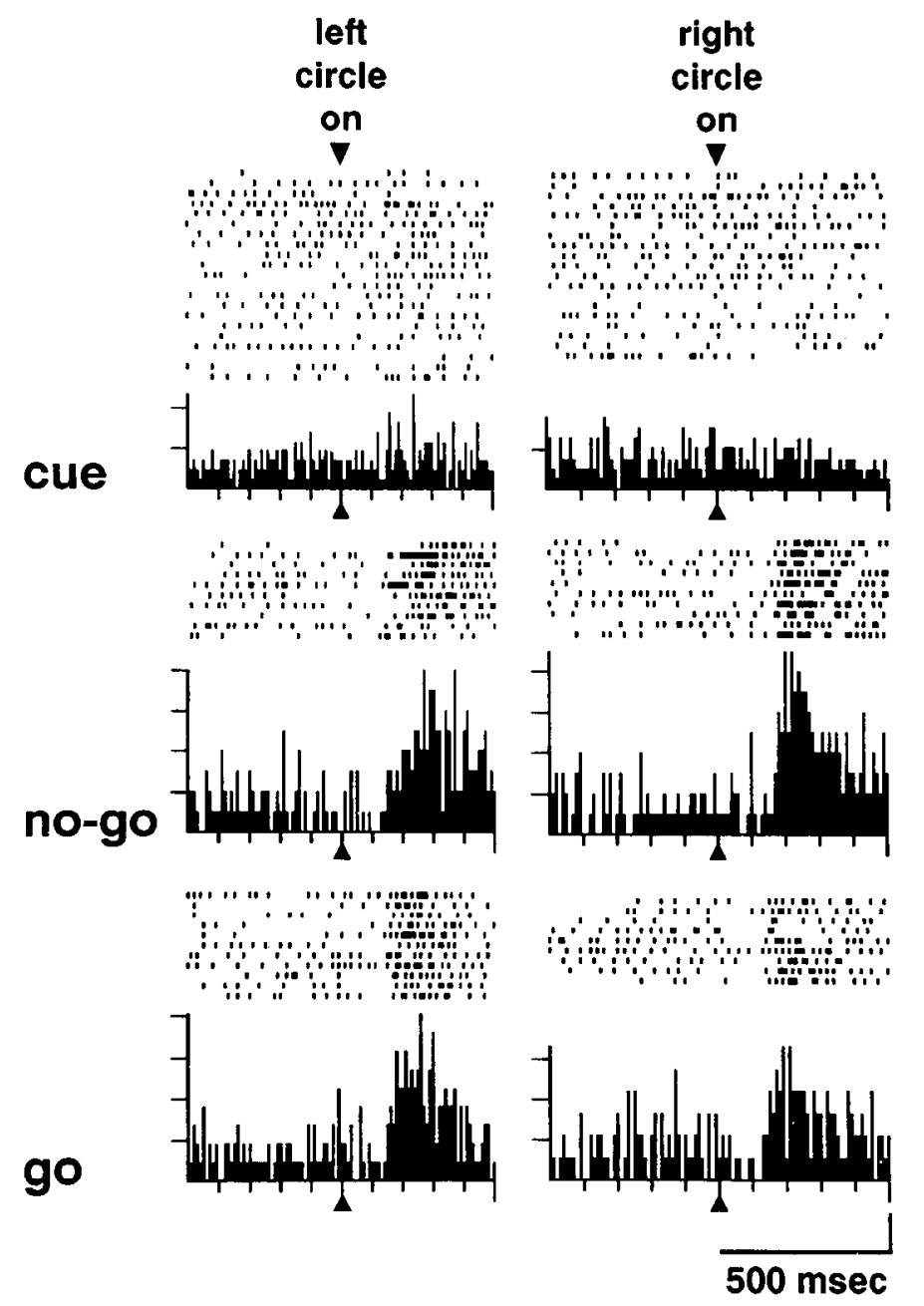

Figure 4. Responses of a basalis neuron in the cue and choice phases of the task. Each dot in the raster represents one discharge of the neuron, and each line of dots is from a single trial. The histograms are generated by collapsing the rasters into $10 \mathrm{msec}$ bins. Each raster and histogram is aligned on the onset of the side circle, which is represented by the solid triangle. The horizontal bar is $500 \mathrm{msec}$ and the vertical bar is 20 spikes/sec. The neuron was located in the anterolateral nucleus basalis. It had no response to the presentation of the side circle in the cue phase but had an increase in firing of 15.5 spikes/ $/ \mathrm{sec}$ when the same stimulus was presented in the no-go or go condition of the choice phase. The increases in firing were not significantly different in the no-go and go conditions although the monkey made arm movements only in the go condition. There were also no differences between the neuronal responses when the circle appeared on the left or right sides of the display screen.

Figure 6 shows the distribution of neuronal response latencies in the go and no-go conditions. The median onset latency was $133 \mathrm{msec}(n=198)$ in the go condition, and $136 \mathrm{msec}(n=201)$ in the no-go condition. These values were slightly higher than the median latency of $124 \mathrm{msec}$ for responses in the choice phase (see Table 2) because both go and no-go trials were used to calculate the latency in the choice phase. When only go trials or only no-go trials were used, the standard deviation in the prestimulus period increased, thereby raising the threshold for response detection, and the higher thresholds delayed the times at which neuronal responses were detected. For the 178 neurons in which responses were detected in both go and no-go condi-

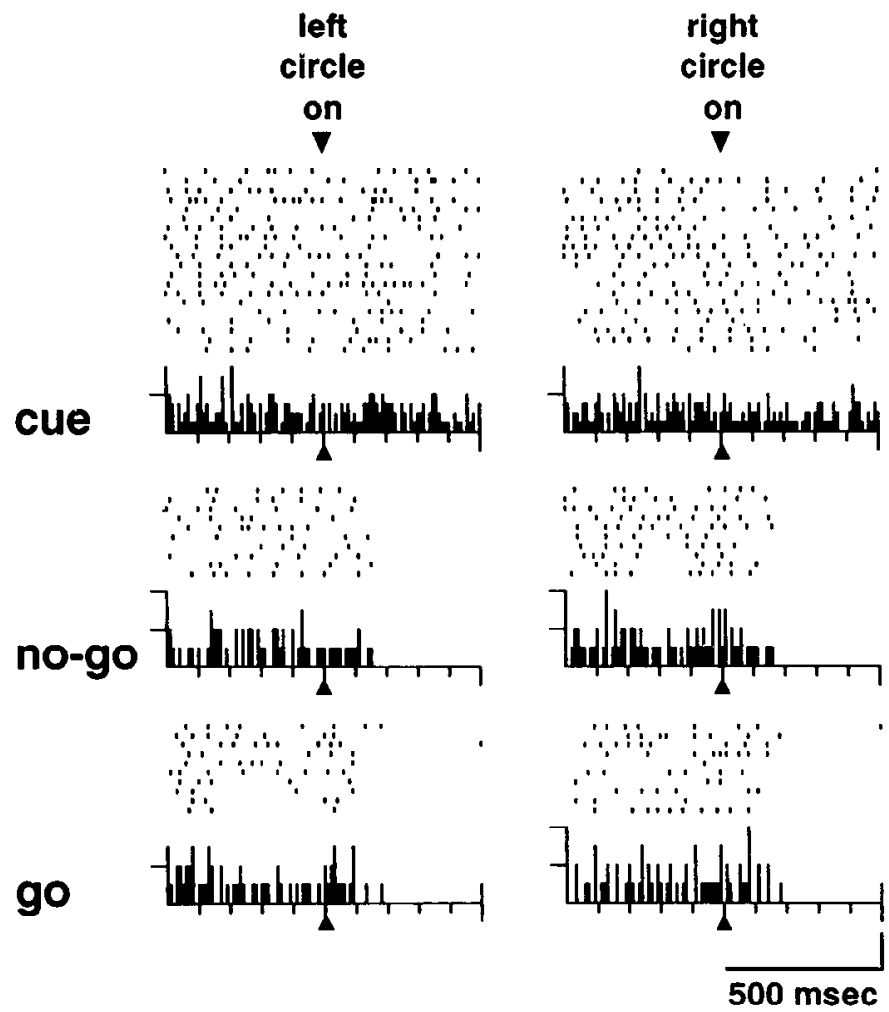

Figure 5. Responses of a basalis neuron in the cue and choice phases of the task. Conventions are the same as in Figure 4. The neuron was from the intermediate division of the nucleus basalis. It had no response in the cue phase but had a sharp decrease in firing of -6.8 spikes/sec in the choice phase. The magnitudes of the decreases in discharge rate were not significantly different in the go and no-go conditions.

tions, the difference between the response latencies was not significant (paired $t=1.56, d f=177, p>0.05$ ).

\section{Cue vs, no-go responses}

Neuronal responses in the no-go condition were compared to responses in the cue condition of the task to determine whether basalis neurons had purely sensory responses. In both conditions, a circle appeared on the left or right side of the screen, and no movement was made by the animal. (The go condition of the choice phase was not used for this comparison since arm movements occurred in it but not in the cue phase.) A one-way ANOVA was performed for all neurons that had significant responses in the cue phase or choice phase of the task. In a sample of 251 neurons, $147(59 \%)$ had significant differences in response magnitude in the cue and no-go conditions. In only one neuron was the response magnitude larger in the cue condition than in the no-go condition. In all other 146 neurons, the response magnitude was larger in the no-go condition. The neurons whose activities are shown in Figures 4 and 5 had significantly larger responses in the no-go condition than in the cue condition.

\section{Neuronal responses in the delay period}

Changes in discharge rates during the 4-6 sec delay period either remained constant or changed steadily throughout the period. Hence, the final $500 \mathrm{msec}$ of the delay period gave a reasonable estimate of the neuron's largest change in activity in that phase of the task. Significant changes in discharge rate from the rate 
immediately before the cue phase were present in $109(33 \%)$ of the task-related neurons. Examples of neuronal responses in the delay period are shown in Figure 7.

A one-way ANOVA was used to determine whether the delay period activity was different depending on whether the side circle appeared on the left or on the right in the cue phase. In only 2 of the 109 neurons was there a significant $(p<0.01)$ difference between the neuron's response magnitude following a cue on one side or the other. A similar analysis was used to detect neuronal responses in the cue or choice phases that reflected the position of the side circle. None of the 18 neurons responding in the cue phase had changes in firing that were significantly larger when the circle appeared on one side or the other. Only 7 (3\%) of the 232 neurons responding in the choice phasc had larger changes in activity depending on whether the side circle appeared on the left or right.

\section{Neuronal responses in the reward phase}

Neuronal responses following the delivery of the water reward were present in $81(25 \%)$ of the task-related neurons. Water was delivered to the animals through a solenoid valve which made a click sound when it opened. In the first animal, the click was audible, but in the second animal, the click was eliminated by placing the valve inside a soundproof container. In the first animal, $32 \%$ of the task-related neurons responded in the reward phase compared to $20 \%$ in the second animal. $T$-test analyses showed that the magnitudes $(t=0.32, d f=78, p>0.05)$ and latencies $(t=-0.51, d f=70, p>0.05)$ of the neuronal responses in the reward phase did not differ significantly between the 2 animals. Examples of neuronal responses in the reward phase are shown in Figure 8.

\section{Regional response differences}

Of the 425 sampled neurons, $54 \%$ were recorded while the monkeys used their ipsilateral arms to perform the task (16\% from the first animal and 38\% from the second animal) and $46 \%$ were recorded while the monkeys used their contralateral arms (31\% from the first animal and 15\% from the second animal). There were no significant differences $(p<0.01)$ by chi-square analysis in the distribution of responses between neurons recorded with either arm. Hence, the arm used appeared to have little influence on the responses of basalis neurons.

Neurons were sampled in 5 divisions of the nucleus basalis: anteromedial, anterolateral, intermediate, posterior, and border regions (the medullary laminae and the anterior commissure). Each division contained neurons that responded in each phase of the GNG task, and there were no significant differences among the divisions in the proportions of responsive neurons in each phase. In the delay period, there was some evidence of an increased number of neurons in the anteromedial and anterolateral divisions showing excitatory responses and a decreased number of neurons in the intermediate and posterior divisions showing excitatory responses $\left(\chi^{2}=17.2, d f=8, p<0.05\right)$.

As shown in Table 1, significant differences in the spontaneous discharge rates of neurons in the 5 divisions were found (oneway ANOVA, $F=4.49, d f=4,418, p<0.01$ ). The lowest discharge rates were in the anteromedial neurons (mean $=17.5$ spikes/sec) and the highest rates were in "border" neurons (mean $=24.1$ spikes/sec). Border neurons also had the highest variability in their discharge patterns, with a mean interspike interval coefficient of variation of $71.1 \%$ compared to $62.6 \%$ for all other basalis neurons $(F=3.57, d f=4,418, p<0.01)$.
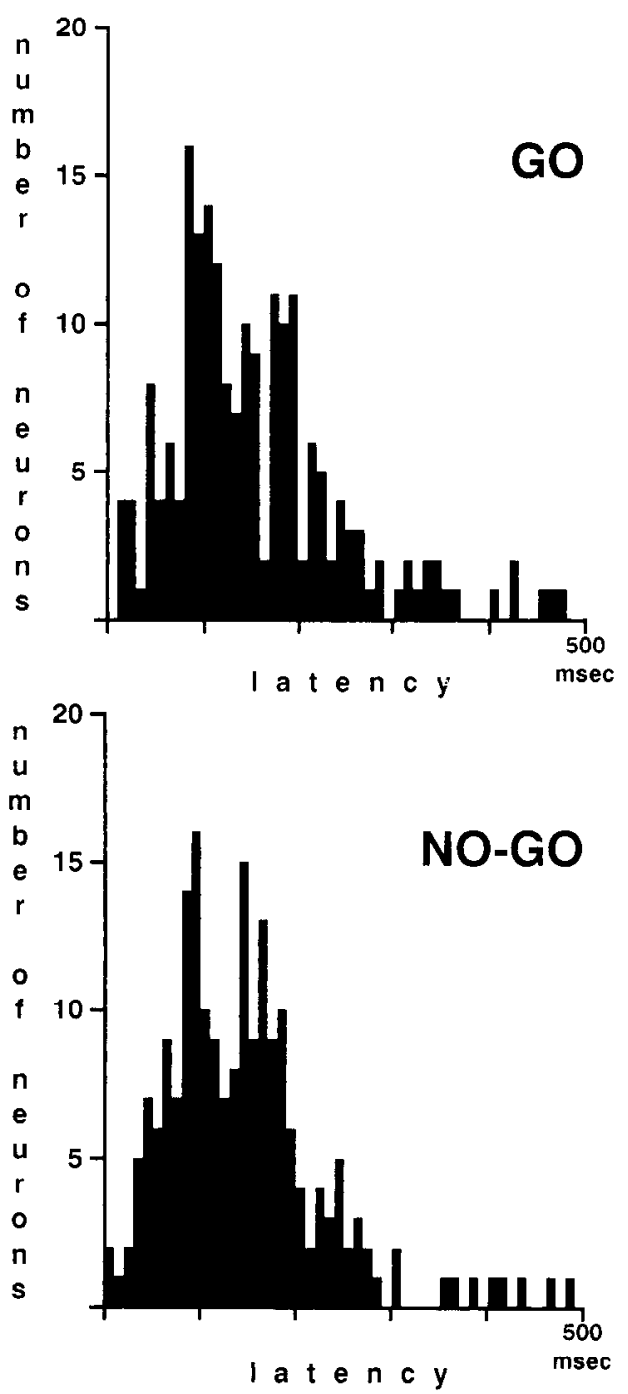

Figure 6. Neuronal response latencies in the go and no-go conditions. The median latencies were $133 \mathrm{msec}$ in the go condition $(n=198)$ and $136 \mathrm{msec}$ in the no-go condition $(n=201)$. The difference between the latencies in the go and no-go conditions was not significant. The latency detection method found 27 neurons with latencies of $50 \mathrm{msec}$ or less. In most cases, such short latencies appeared to result from chance variations in firing or shifts in baseline firing just before the onset of the neuronal response, as determined visually.

\section{Error trials}

Incorrect trials were analyzed for the 228 neurons that responded in the choice phase. Errors occurred on approximately $11 \%$ of the trials in this group, and in $6 \%$ of all trials, the errors occurred in the choice phase of the task. Almost all $(92 \%)$ of the errors in the choice phase occurred in the go condition, when the monkey either did not move his arm at all, did not move in the correct direction, or did not move accurately within 650 msec. The remaining $8 \%$ of the errors occurred when the monkey moved his arm in the no-go condition.

Because of the small number of trials on which errors were made, it was impractical to analyze the neuronal activity on error trials statistically. However, it was possible to qualitatively evaluate changes in activity in some cells when several error trials occurred and the neuronal responses were robust, as shown in Figure 9. In 51 of 52 neurons that had clear responses on 

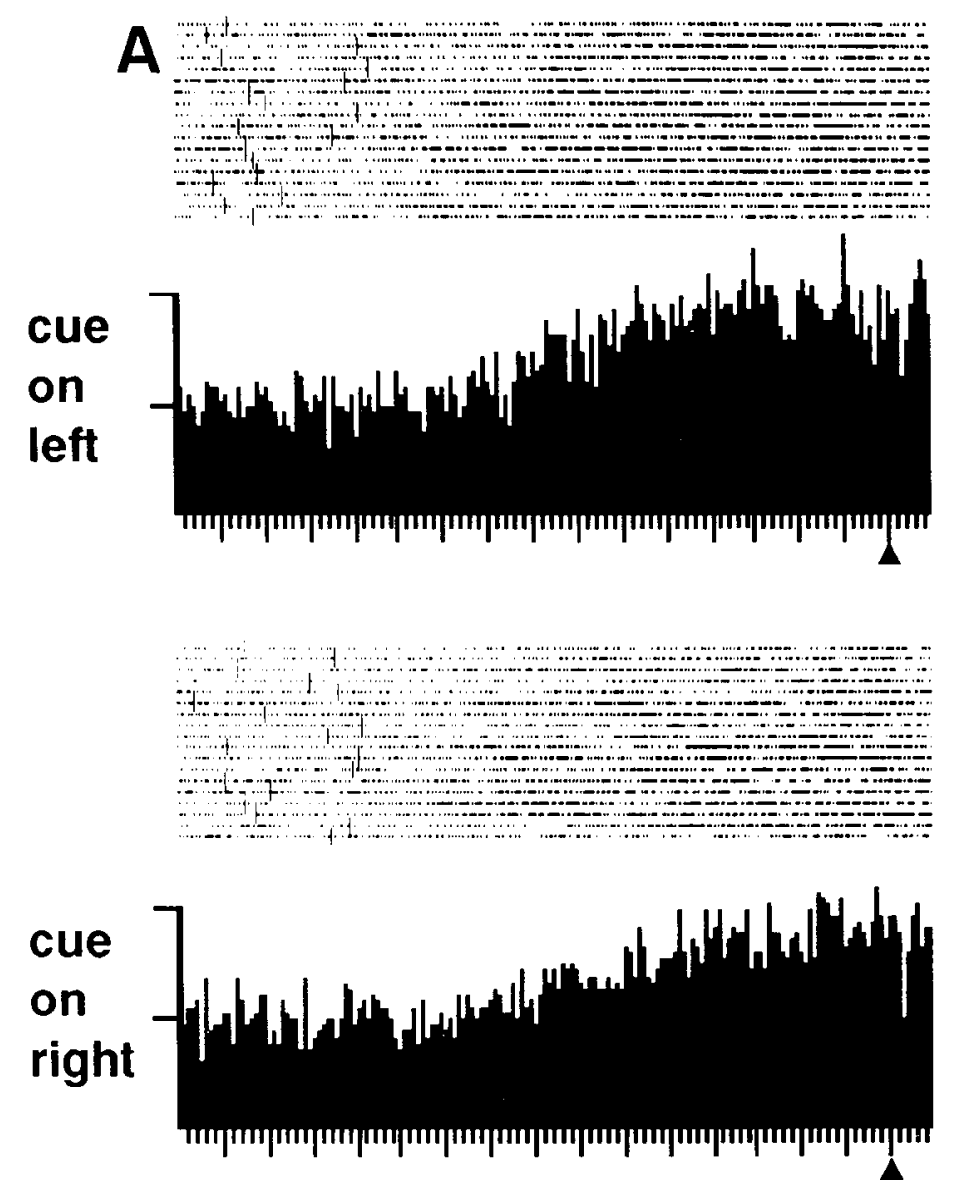

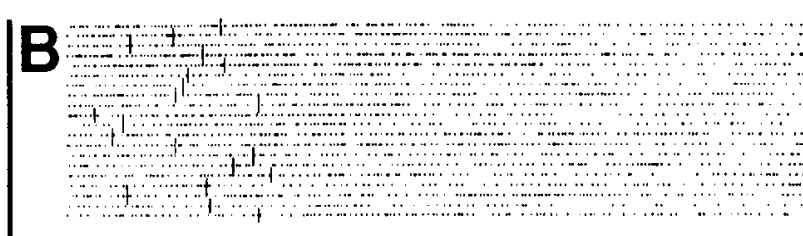

(1)
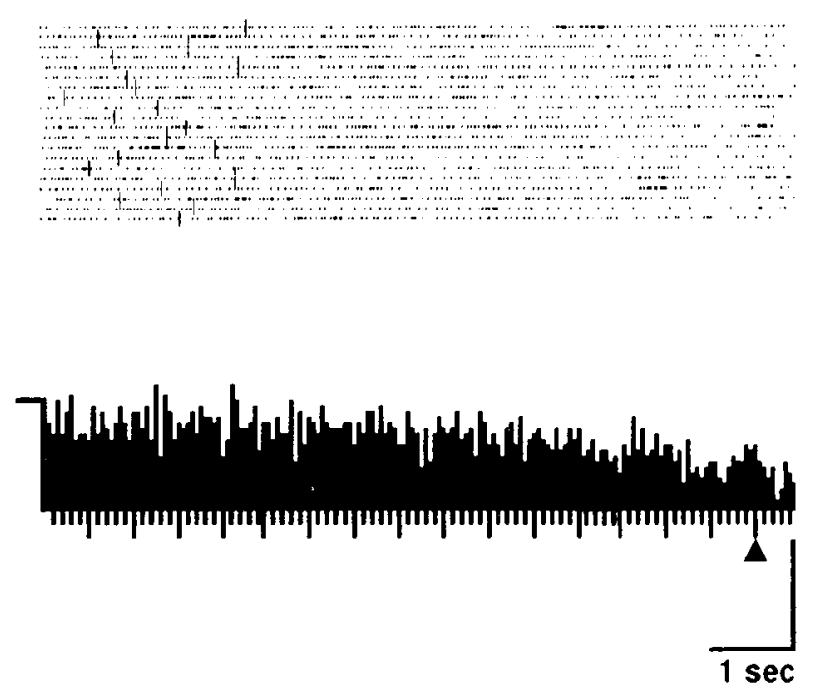

Figure 7. Responses of basalis neurons in the delay period. Conventions are the same as in Figure 4 except that $50 \mathrm{msec}$ bins are used in the histograms. The short vertical bars on the left side of the rasters mark the onset of the side circle in the cue phase, and the triangles on the histograms mark the onset of the side circle in the choice phase. The neuron in $A$ was located in the intermediate nucleus basalis and showed an increase in discharge rate of $14.9 \mathrm{spikes} / \mathrm{sec}$ in the final $500 \mathrm{msec}$ of the delay period for all trials combined. The neuron in $B$ was in the anterolateral nucleus basalis and had a decrease in discharge rate of $-7.7 \mathrm{spikes} / \mathrm{sec}$. For both neurons, there was no difference between the magnitude of the changes in firing when the circle in the preceding cue phase appeared on the left or the right side of the display screen.

error trials, the polarity of the response (increase or decrease) in the choice phase on error trials was the same as on correct trials. Moreover, in $43(80 \%)$ of 54 neurons that were recorded on 5 or more trials with choice errors, changes in discharge rate on error trials appeared to be equal to the responses on correct trials. The remaining 11 neurons had no clear changes in firing in the choice phase on error trials. Thus, to the extent that the data could be analyzed, it appeared that neuronal responses on error trials were largely the same as the responses on correct trials.

\section{Discussion}

This study was designed to determine whether the changes in activity of basalis neurons in a behavioral task arc primarily related to perceiving a stimulus, generating a movement, or neither. If the activity of hasalis neurons were directly related to perceiving a stimulus, then the changes in discharge rate in response to the sensory stimulus would be similar each time the animal perceived that stimulus. However, in the GNG task, the changes in neuronal firing were much more prevalent and of greater magnitude when the side circle stimulus appeared in the choice phase than when the same stimulus appeared in the cue phase. Therefore, changes in activity of most basalis neurons are unlikely to be directly related to the perception of the visual stimulus.

If the activity of basalis neurons were related to the execution of a movement, then the changes in discharge rate associated with a movement would be most prominent when that movement occurred. However, for most basalis neurons in the GNG task, the change in neuronal firing in the choice phase when a movement was made was equivalent to the change in firing when no movement was made. Therefore, the changes in activity of most basalis neurons could not be directly related to the execution of a movement.

\section{Relation to movement}

Of 253 basalis neurons that responded in the choice phase, $79 \%$ had essentially the same change in discharge rate regardless of whether the animal made an arm movement or remained motionless. Another $9 \%$ of the neurons had larger responses when 

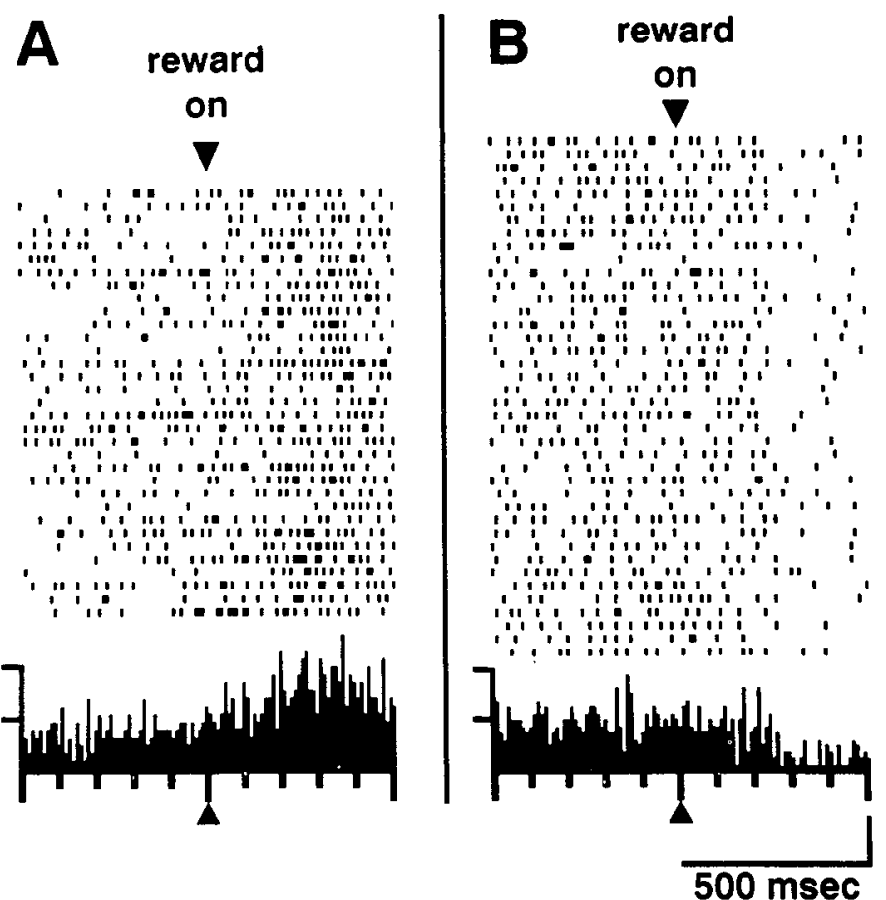

Figure 8 . Responses of basalis neurons in the reward phase. Conventions are the same as in Figure 4. The triangles indicate the time of the opening of the solenoid valve to dispense the water reward. All 4 trial types are combined since the neuronal responses were the same on each one. The neuron represented in $A$ was from the anterolateral nucleus basalis and had an increase in firing of $14.6 \mathrm{spikes} / \mathrm{sec}$ in the reward phase. The neuron in $B$ was from the anteromedial nucleus basalis and had a decrease in firing of -7.2 spikes $/ \mathrm{sec}$.

the animal did not move than when he did. Recordings of EMG activity confirmed that there was little, if any, contraction of the elbow flexors or extensors in the no-go condition. Thus, the responses of most basalis neurons in the choice phase were most likely related to some process other than the execution of the arm movement.

Since neuronal responses in the go and no-go conditions were similar, it is conceivable that they were related to the animal's movements to receive the reward. In both the go and no-go conditions, each animal typically extended its tongue, lips, and head toward the water spout before the reward delivery. Based on the neck EMG recordings, these preparatory movements began at approximately 1000 msec into the choice phase on nogo trials and $1500 \mathrm{msec}$ on go trials. Although the latencies to the reward preparation movements were different on go and nogo trials, the latencies to the responses of basalis neurons were essentially the same, as demonstrated in Figure 6. Moreover, each animal often licked the spout repeatedly during the trial, but no basalis neurons were found to respond phasically with the licking movement. It is therefore highly unlikely that the robust responses of basalis neurons in the choice phase were dependent on the animal's movements to receive the reward.

Results from our prior studies also support the conclusion that most basalis neurons do not respond with movement, per se (DeLong, 1971; Richardson and DeLong, 1986b). In a DR task, basalis neurons often had larger changes in activity when the monkey made an arm movement in the choice phase of the task than when he made the same movement in the cue phase.

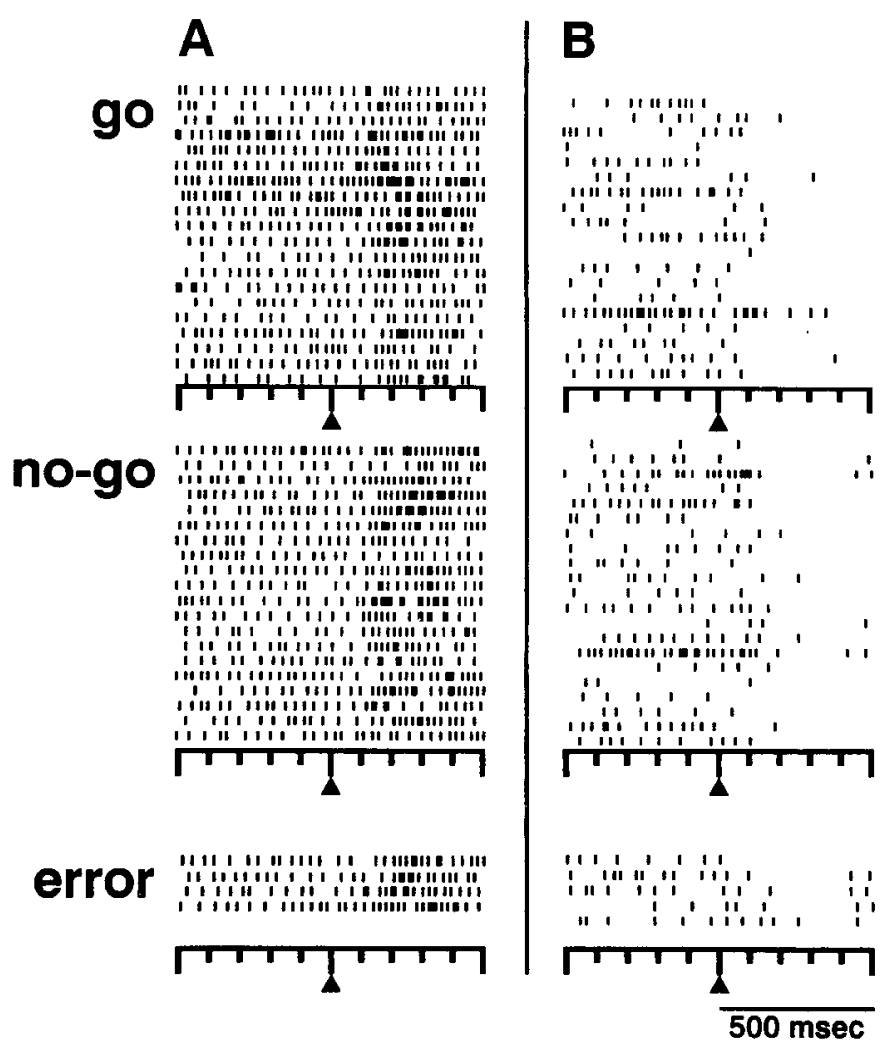

Figure 9. Responses of basalis neurons in the choice phase on error trials. The rasters are aligned on the onset of the visual stimulus in the choice phase, as indicated by the solid triangles. The neuron represented in $A$ was from the anteromedial nucleus basalis and showed an increase in discharge rate of 9.7 spikes/sec on correct go and no-go trials. A similar increase in firing was also apparent on 4 error trials. In $B$, the neuron was from the posterior nucleus basalis and showed a decrease in firing of $-9.3 \mathrm{spikes} / \mathrm{sec}$ in the go and no-go conditions and a similar decrease in firing on 5 error trials. All of the errors resulted from the monkey not moving its arm on go trials, except for the first error trial in $B$, which resulted from a movement in the wrong direction on a go trial.

It therefore appeared that the neuronal responses were strongly influenced by the context in which the movement was made.

\section{Relation to sensory stimuli}

Comparable reasoning indicates that the responses of basalis neurons in the GNG task were not closely associated with the visual stimuli, per se, but were dependent on the context of the stimuli. The same visual stimuli (circles appearing on the left or right sides of the display screen) were presented in the cue and choice phases of the GNG task. In the cue phase, the side circle indicated the position to be remembered by the monkey during the delay period. In the choice phase, the side circle indicated whether the monkey had to move or not move his arm in order to obtain the water reward. Comparisons were made between the neuronal responses in the cue phase and the no-go condition of the choice phase because no arm movements occurred in either condition and the same visual stimuli occurred in both conditions. Hence, the primary difference between the cue and no-go conditions was the significance of the visual stimulus for the animal.

Despite the similarities between the cue and no-go conditions, basalis neurons had strikingly different responses in the 2 con- 
ditions. Changes in discharge rate were much more prevalent and of greater magnitude in the no-go condition than in the cue condition. In a sample of 251 neurons that responded in the cue or choice phases, close to $60 \%$ had significantly larger responses in the no-go condition than in the cue condition. Moreover, only $5 \%$ of all task-related basalis cells had detectable changes in activity in the cue phase, whereas $70 \%$ had detectable changes in the choice phase. Thus, the responses of most basalis neurons in the choice phase were probably not directly related to the perception of the visual stimulus.

Although the responses of basalis neurons in the GNG task do not appear to be contingent on the perception of a specific stimulus or the execution of a specific movement, they are clearly highly contingent on some aspect of the choice phase. The exact conditions that lead to the frequent, robust ncuronal responses in the choice phase are not completely understood. Currently, 2 possibilities appear to be the most likely. The neuronal responses in the choice phase may be related to the decision-making processes used by the animals to choose accurately, or they may be related to the arousal properties of the choice phase since it consistently precedes the water reward.

\section{Relation to decision-making processes}

When the side circle appeared at the onset of the choice phase, the animal had to decide within a few hundred milliseconds whether to move or remain motionless in order to obtain a water reward. It is possible that the changes in discharge rates of basalis neurons in the choice phase formed an integral part of the neural substrate underlying that decision process. The decision to move or not move had to occur within the first 230 msec of the choice phase because increases in arm EMG activity began at approximately $230 \mathrm{msec}$ after the start of the choice phase. Significant changes in the discharge rates of basalis neurons in the choice phase had a median latency of $124 \mathrm{msec}$, and $90 \%$ of the neuronal responses in the choice phase began before the increase in EMG activity at $230 \mathrm{msec}$. Hence, the changes in activity of basalis neurons were early enough to be involved in the decision process leading to correct behavioral responses in the choice phase.

Since neuronal response magnitudes and latencies were similar in the go and no-go conditions, any decision process in which the nucleus basalis may be involved must be similar on go and no-go trials. That is, basalis neurons would not have been selectively involved in either the decision to move or the decision not to move, but they would have been involved in some aspect of the decision process that was common to both the go and no-go conditions.

It is important to consider various ways in which the monkeys might have performed the GNG task. An animal could have used at least 2 strategies. The first strategy would have been for the animal to decide whether the circle presented in the choice phase was on the same or opposite side of the circle presented in the preceding cue phase. If it were on the same side, the animal would have decided to move the dot to the circle by moving his arm, but if it were on the opposite side, he would have decided to keep his arm motionless. Thus, a distinct decision would have been made in both go and no-go conditions.

A second possible strategy would have been for the animal to use the side circle in the cue phase as a target for the movement in the subsequent choice phase. If the "cued" circle appeared again in the choice phase, the animal would have moved to it. Alternatively, if the "cued" circle did not appear in the choice phase as on no-go trials, there would be no stimulus to move, and the animal would remain motionless. Thus, the correct behavioral response in the no-go condition would not have resulted from a decision, but rather from a lack of a triggering stimulus for the movement.

If the animals used the first strategy, in which a decision is made in both go and no-go conditions, then the responses of basalis neurons in the choice phase could have possibly reflected the decision process. Conversely, if the animals used the second strategy, in which the decision process in the go condition is quite different from the no-go condition, then it is unlikely that the responses of basalis neurons were related to the decision process.

It is often useful to study error trials to determine whether changes in neuronal activity reflect the animal's incorrect behavior. Unfortunately, analysis of error trials in the present study provided little insight to whether the neuronal responses could have been related to decision-making processes. Errors in the choice phase resulted when the animal (1) moved on a no-go trial, (2) did not move on a go trial, (3) moved to the wrong side on a go trial, (4) moved inaccurately to the correct side on a go trial. In all 4 cases, it was possible that the animal made a decision, although it could have been an incorrect decision. In many other behavioral tasks, the lack of a movement in the choice phase would most likely result from the lack of the animal making a decision. However, in the GNG task, "no movement" could have resulted from the animal deciding not to move rather than the animal making no decision at all. Thus, there was no condition in which the animal clearly did not make a decision. Further studies in which decision and nondecision conditions can be dissociated are necessary to determine whether the activity of nucleus basalis neurons is related to decisionmaking processes.

Changes in neuronal activity in the choice phase on error trials appeared to be largely the same as the responses on correct trials. If the neuronal responses were related to decision-making processes, then the same decision process must have been occurring on both correct and incorrect trials. Therefore, when no movement occurred on go trials, the animal had to be deciding not to move according to the decision-making hypothesis. Although a decision-related interpretation of the responses of basalis neurons cannot be ruled out, it is tenable only if the absence of movement on both no-go trials and error trials resulted from a decision not to move rather than the absence of a decision to move.

\section{Relation to stimuli associated with rewards}

Although the responses of basalis neurons in the choice phase of the GNG task may have been related to decision-making processes, there are several conditions in which basalis neurons have robust responses when no decision is required of the animal. In particular, basalis neurons are often quite responsive to stimuli that consistently precede a reward or to the reward itself.

One of the earliest and most consistent findings regarding basalis neurons has been that they often respond to a water reward delivered at the end of correct trials in complex behavioral tasks (DeLong, 1971; Richardson et al., 1988). A large proportion of basalis neurons also respond to water delivered outside the context of a behavioral task (Richardson and DeLong, 1990). In the present study, a much smaller proportion (25\%) of basalis neurons responded following the reward delivery than 
in previous studies, in which at least $44 \%$ of the neurons responded in the reward phase. One contributing factor to the reduced percentage of neurons responding in the reward phase may have been that the solenoid valve which dispensed the water was inside a soundproof container when recordings were made in the second animal. However, the first animal was able to hear the solenoid valve opening, and only $32 \%$ of the basalis neurons responded in the reward phase in that animal. Other possible explanations are discussed below.

Basalis neurons have been found to respond not only to water rewards, but also to stimuli that consistently precede rewards. For example, we have found that over half of the basalis neurons that responded to simple somatosensory or auditory stimuli had larger changes in firing to a stimulus that consistently preceded a water reward compared to a stimulus of the same modality that did not precede a reward (unpublished observation). Moreover, in previous studies using a visuomotor tracking task and a delayed response task, basalis neurons responded most often in the phase of the task that immediately preceded the delivery of a water reward (Richardson and DeLong, 1986b; Mitchell et al., 1987).

Similar responses were observed in basal forebrain neurons recorded by Rolls and associates (Burton et al., 1976; Mora et al., 1976; Rolls et al., 1976, 1979). They studied neurons that responded selectively to the sight of food objects and visual stimuli that monkeys had learned to associate with food or juice. In certain neurons, if the visual stimulus was no longer followed by a food reward, the neuronal response extinguished. In addition, the neuronal responses did not appear to be related to specific movements of the monkey. Although their earlier conclusions were based on a relatively small number of neurons, some of which were located in the lateral hypothalamus, their more recent studies, based on substantially larger populations of neurons, have also shown that basalis neurons respond selectively to visual stimuli associated with appetitive rewards (Wilson et al., 1984).

Rigdon and Pirch (1986) have reported comparable findings in their study of neuronal activity in the basal forebrain of rats. They found that a high proportion of neurons (28/38) responded to a tone that consistently preceded electrical stimulation of the medial forebrain bundle (MFB). MFB stimulation was considered to be a reinforcement because it supported self-stimulation in the rats. Moreover, if the MFB stimulation was not given after the tone cue, the responses to the tone extinguished.

In the present study, basalis neurons were clearly most responsive in the choice phase of the GNG task, and they were, in gencral, cqually responsive in the go condition and the nogo condition. In approximately $94 \%$ of the trials, the choice phase immediately preceded the reward delivery by a few seconds. Since basalis neurons appear to be particularly responsive to stimuli that precede rewards, their responses in the choice phase may have resulted from the fact that the choice phase consistently preceded the reward in well-trained animals.

The responses of basalis neurons in the choice phase were most likely not related to the animal's expectation of the water reward as indicated by its behavioral responses. Prior to when the animal expected the reward, it extended its tongue and head toward the water spout. As discussed earlier, the behavioral responses had markedly different latencies on go and no-go trials, whereas the neuronal responses had essentially the same latencies. Moreover, neuronal responses in the choice phase always ended before the reward delivery. Hence, the changes in neuronal activity at the beginning of the choice phase were no longer present when the animal clearly expected to receive the reward. Therefore, the responses of basalis neurons did not appear to be related to reward expectation.

The timing of the neuronal responses in the choice phase suggested that they were triggered by the appearance of the side circle. The responses of basalis neurons did not appear to be directly related to the execution of an arm movement because similar responses occurred when there was no movement. Furthermore, the onsets of the neuronal responses on go trials rarely appeared to be better aligned with the arm movement than with the appearance of the side circle. Therefore, basalis neurons appeared to respond to the visual stimulus when it occurred in close temporal relation to the reward.

\section{Relation to arousal}

As mentioned earlier, previous studies suggested that nucleus basalis neurons are highly responsive to stimuli associated with rewards. The functional significance of the reward-related responses remains uncertain, but one possibility is that they may be related to arousal processes rather than reinforcement (Richardson and DeLong, 1988). Numerous observations indicate that the nucleus basalis plays a role in the cortical activation component of arousal. Cholinergic agonists have long been known to induce cortical EEG desynchronization, whereas antagonists induce synchronization (see Defeudis, 1974). During periods of cortical desynchronization, there is a strong increase in the baseline firing rates of basalis neurons (Szymusiak and McGinty, 1986; Detari and Vanderwolf, 1987) and the release of ACh in cortex (Pepeu, 1974; Rasmusson and Szerb, 1976). Lesions in the basal forebrain also reduce high-frequency components of the cortical EEG that correspond to increased levels of arousal (Stewart et al., 1984; Buzsaki et al., 1988). Finally, the morphology and distribution of basalis neurons (Das and Kreutzberg, 1968) and their anatomical connections with cerebral cortex (Shute and Lewis, 1967), brain stem (Semba et al., 1988; Vertes, 1988), and the reticular thalamic nucleus (Levey et al., 1987; Steriade et al., 1987) have led many researchers to consider the nucleus basalis to play a key role in cortical activation.

It therefore appears that changes in the baseline firing rates of basalis neurons reflect changes in an animal's level of arousal. Phasic changes in discharge rate lasting a few hundred milliseconds may therefore reflect brief changes in arousal. It is possible that basalis neurons respond to stimuli associated with rewards because such stimuli are particularly arousing to the animal. Hence, in the GNG task, basalis neurons may have becn most responsive in the choicc phase bccausc it may have been the most arousing part of the task. In the choice phase, the monkey had to quickly decide whether or not to make a rapid movement, and if it performed correctly, it always received a water reward. It is possible that the reward itself became less arousing to the animal as it learned that the reward was contingent on accurate performance in the choice phase. This possibility could account for the relatively small percentage of basalis neurons that responded in the reward phase of the task. Previous observations have also implied that the responses of basalis neurons to a reward may be diminished when the reward is consistently preceded by another stimulus (Richardson and DeLong, 1990).

The interpretation that changes in the discharge rates of basalis neurons are associated with increased levels of arousal may also explain why there was an increase in the percentage of 
neurons responding in the delay period of the GNG task (33\%) compared to the previous DR task (14\%). In both tasks, very few neurons had differential responses reflecting the position of the side circle in the preceding cue phase. The lack of differential responses implies that the nucleus basalis was probably not directly involved in the memory processes required to remember the position of the side circle during the delay period. Nevertheless, the finding that there was a higher percentage of basalis neurons responding in the delay period of the GNG task may be related to the increased task difficulty. The GNG task had 4 types of trials and 3 types of behavioral responses, whereas the DR task had only 2 types of trials and 2 types of behavioral responses. The increased difficulty of the GNG task may have been associated with higher levels of arousal, which may have been reflected in the increased percentage of basalis neurons responding in the delay period.

The hypothesis that the phasic responses of basalis neurons reflect brief changes in arousal levels is compatible with the finding that the tonic firing patterns of basalis neurons correlate with changes in cortical activation (Szymusiak and McGinty, 1986; Detari and Vanderwolf, 1987). Nevertheless, there must be an independent measure of changes in arousal levels within the short time frame of the phasic responses (approximately 500 msec) in order to test the hypothesis. Most measures of arousal, such as heart rate, respiration rate, or galvanic skin response, change too slowly to be useful for this purpose. Changes in EEG activity from a synchronized to a desynchronized state can be detected over a short time period, but the EEG would probably be continually desynchronized in animals performing behavioral tasks. Further studies are needed to determine whether brief changes in EEG activity can be detected in behaving monkeys and whether such changes correlate with changes in the activity of nucleus basalis neurons.

\section{Summary}

Neurons in the nucleus basalis were most responsive in the choice phase of a go/no-go task in monkeys. In the choice phase, the animal had to view a visual stimulus and quickly decide whether or not to make a specific arm movement in order to obtain a water reward. The responses of basalis neurons in the choice phase did not appear to be due to the arm movement because similar responses occurred when the animal made no movement. The neuronal responses also did not appear to be due to the visual stimulus per se because there were rarely responses to the same stimulus presented earlier in the cue phase of the task. The changes in neuronal activity in the nucleus basalis, therefore, appeared to be largely dependent on the context of the stimulus. The neuronal responses in the choice phase were possibly related to the decision-making processes. However, based on the findings of previous studies, the responses of basalis neurons were more likely related to a transient increase in arousal triggered by the visual stimulus in the choice phase. The stimulus is hypothesized to be arousing because it consistently preceded the water reward.

\section{References}

Burton MJ, Rolls ET, Mora F (1976) Effects of hunger on the responses of neurons in the lateral hypothalamus to the sight and taste of food. Exp Neurol 51:668-677.

Buzsaki G, Bickford RG, Ponomareff G, Thal IJ, Mandel R, Gage F (1988) Nucleus basalis and thalamic control of neocortical activity in the freely moving rat. J Neurosci 8(11):4007-4026.
Collerton D (1986) Cholinergic function and intellectual decline in Alzheimer's disease. Neuroscience 19(1):1-28.

Das GD, Kreutzberg GW (1968) Evaluation of interstitial nerve cells in the central nervous system: a correlative study using acetylcholinesterase and Golgi techniques. Ergeb Anat Entwickl Gesch 41:1-58.

Defeudis FV (1974) Cholinergic roles in consciousness. In: Central cholinergic systems and behaviour, pp 7-32. New York: Academic.

DeLong MR (1971) Activity of pallidal neurons during movement. J Neurophysiol 34:414-427.

Detari L, Vanderwolf CH (1987) Activity of identified cortically projecting and other basal forebrain neurones during large slow waves and cortical activation in anaesthetized rats. Brain Res 437:1-8.

Fuster JM (1973) Unit activity in prefrontal cortex during delayedresponse performance: neuronal correlates of transient memory. $J$ Neurophysiol 36:61-78.

Kimball AW (1987) A test for a threshold in an ordered sequence of correlated proportions. Biometrics 43:707-712.

Kojima S, Guldman-Rakic PS (1982) Delay-related activity of prefrontal neurons in rhesus monkeys performing delayed response. Brain Res 248:43-49.

Levey AI, Hallanger AE, Wainer BH (1987) Cholinergic nucleus basalis neurons may influence the cortex via the thalamus. Neurosci Lett 74:7-13.

Mesulam M, Mufson EJ, Levey AI, Wainer BH (1983) Cholinergic innervation of cortex by the basal forebrain: cytochemistry and cortical connections of the septal area, diagonal band nuclei, nucleus basalis (substantia innominata), and hypothalamus in the rhesus monkey. J Comp Neurol 214:170-197.

Mesulam M, Mufson EJ, Wainer BH (1986) Three dimensional representation and cortical projection topography of the nucleus basalis (Ch4) in the macaque: concurrent demonstration of choline acetyltransferase and retrograde transport with a stabilized tetramethylbenzidine method for horseradish peroxidase. Brain Res 367:301308.

Mitchell SJ, Richardson RT, Baker FH, DeLong MR (1987) The primate nucleus basalis of Meynert: neuronal activity related to a visuomotor tracking task. Exp Brain Res 68(3):506-515.

Mora F, Rolls ET, Burton MJ (1976) Modulation during learning of the responses of neurons in the lateral hypothalamus to the sight of food. Exp Neurol 53:508-519.

Niki H, Watanabe M (1976) Prefrontal unit activity and delayed response. Relation to cue location versus direction of response. Brain Res 105:79-88.

Olton DS, Wenk GL (1987) Dementia: animal models of the cognitive impairments produced by degeneration of the basal forebrain cholinergic system. In: Psychopharmacology: the third generation of progress (Meltzer HY, ed), pp 941-953. New York: Raven.

Pepeu G (1974) The release of acetylcholine from the brain: an approach to the study of the central cholinergic mechanisms. Prog Neurobiol 2:257-288.

Rasmusson D, Szerb JC (1976) Acetylcholine release from visual and sensorimotor cortices of conditioned rabbits: the effects of sensory cuing and patterns of responding. Brain Res 104:243-259.

Richardson RT, DeLong MR (1986a) Differential responses of nucleus basalis of Meynert neurons in a go/no-go task in monkey (Macaca mulatta). Soc Neurosci Abstr 12:356.

Richardson RT, DeLong MR (1986b) Nucleus basalis of Meynert neuronal activity during a delayed response task in monkey. Brain Res 399:364-368.

Richardson RT, DeLong MR (1987) Tonically active nucleus basalis neurons in the awake monkey project to cerebral cortex. Soc Neurosci Abstr 13:1027.

Richardson RT, DeLong MR (1988) A reappraisal of the functions of the nucleus basalis of Meynert. Trends Neurosci 11(6):264-267.

Richardson RT, DeLong MR (1990) Responses of primate nucleus basalis neurons to water rewards and related stimuli. In: Brain cholinergic systems (Steriade M, Biesold D, eds). New York: Oxford, pp 282-293.

Richardson RT, Mitchell SJ, Baker FH, DeLong MR (1988) Responses of nucleus basalis of Meynert neurons in behaving monkeys. In: Cellular mechanisms of conditioning and behavioral plasticity (Woody CD, Alkon DL, McGaugh JL, eds), pp 161-173. New York: Plenum.

Rigdon GC, Pirch JH (1986) Nucleus basalis involvement in conditioned neuronal responses in the rat frontal cortex. J Neurosci 6(9): $2535-2542$. 
Rolls ET, Burton MJ, Mora F (1976) Hypothalamic neuronal responses associated with the sight of food. Brain Res 111:53-66.

Rolls ET, Sanghera MK, Roper-Hall A (1979) The latency of activation of neurones in the lateral hypothalamus and substantia innominata during feeding in the monkey. Brain Res 164:121-135.

Semba K, Reiner PB, McGeer EG, Fibiger HC (1988) Brainstem afferents to the magnocellular basal forebrain studied by axonal transport, immunohistochemistry and electrophysiology in the rat. J Comp Neurol 267:433-453.

Shute CCD, Lewis PR (1967) The ascending cholinergic reticular system: neocortical, olfactory, and subcortical projections. Brain 90:497519.

Steriade M, Parent A, Pare D, Smith Y (1987) Cholinergic and noncholinergic neurons of cat basal forebrain project to reticular and mediodorsal thalamic nuclei. Brain Res 408:372-376.
Stewart DJ, Macfabe DF, Vanderwolf CH (1984) Cholinergic activation of the electrocorticogram: role of the substantia innominata and effects of atropine and quinuclidinyl benzilate. Brain Res 322: 219-232.

Struble RG, Lehmann JL, Mitchell SJ, McKinney M, Price DL, Coyle JT, DeLong MR (1986) Basal forebrain neurons provide major cholinergic innervation of primate neocortex. Neurosci Lett 66:215220.

Szymusiak R, McGinty D (1986) Sleep-related neuronal discharge in the basal forebrain of cats. Brain Res 370:82-92.

Vertes RP (1988) Brainstem afferents to the basal forebrain in the rat. Neuroscience 24(3):907-935.

Wilson FA, Rolls ET, Yaxley S, Thorpe SJ (1984) Responses of neurons in the basal forebrain of the behaving monkey. Soc Neurosci Abstr 10:128. 